ISSN 1112-9867

\title{
DEVELOPMENT OF AN OBJECTIVE METHOD FOR THE ASSESSMENT OF NYLON SUTURES KNOT SECURITY
}

\author{
S. Turki ${ }^{*}$ and S. Ben Abdessalem \\ Monastir University, National Engineering School of Monastir, Textile Materials and processes \\ Research Unit, 5000, Tunisia
}

Received: 03 November 2016 / Accepted: 24 April 2017 / Published online: 01 May 2017

\begin{abstract}
A perfect wound healing requires sutures having many handling characteristics such as good knot security and flexibility. Knot security characteristics are influenced by a variety of physical and mechanical parameters including the friction coefficient, bending rigidity and compressibility of the suture. Knot security and other handling characteristics are evaluated only by surgeons during the implantation. There are no standard tests to evaluate these qualities. We present in this paper an objective method to assess knot security of nylon sutures. This method is based on a fuzzy-logic model which correlates suture physical parameters and the knot security.
\end{abstract}

Key words: Nylon braided sutures, handling characteristic, knot security, physical parameters, fuzzy-logic model.

Corresponding author; E-mail: samar.turki@hotmail.fr

doi: http://dx.doi.org/10.4314/jfas.v9i2.24

\section{INTRODUCTION}

Sutures are used in all surgical operations and are the most implanted biomaterials. Linen sutures are the first sutures used by Egyptians [1]. There are several types of sutures which present different physical and chemical properties. These sutures can be classified according to their origins: natural or synthetic, according to their behavior in tissue: absorbable or non absorbable and in terms of their physical configurations, suture materials can be classified into monofilaments or multifilaments. 
In order to ensure a perfect and complete healing of a wound, sutures should always meet certain requirements and characteristics. These characteristics can be classified into three types: physiological, physical and handling qualities. Handling characteristics are generally appreciated subjectively by the surgeon. It's the category of suture characteristics related to the 'feel' of suture materials by surgeons during wound healing. These characteristics including pliability, flexibility, knot tie down and knot security are the most difficult to be evaluated objectively. In fact, industrially, only the diameter and the tensile strength of the suture are tested.

Handling characteristics are related to physical and mechanical characteristics [2]. For example, "knot security" which describes the ability of the knot to stay in position without untying or slippage is related to the coefficient of friction, stiffness or bending rigidity, size, compressibility of the suture and tying technique. The present study aims to lay some assessment parameters for handling characteristics.

Multifilament sutures such as braided sutures are generally easier to handle and tie than monofilament sutures. They provide a better knot security due to their higher coefficient of friction [2, 3]. A linear relationship between knot security and coefficient of friction was reported by Herman [4]. A high coefficient of friction makes the knot tying difficult but leads to a more secure knot thanks to additional frictional forces which hold the knot ears together.

Determining the knot security of the sutures is the subject of many researches in orthopedic surgery $[5,6]$. This literature focuses on knot security because it plays an important role in the successful wound healing but reported results are not usually approved clinically. Indeed, there are no conventional procedures for objectively evaluating the handling properties of sutures [7].

We propose, in this paper, an objective method for evaluating knot security based on the correlation between subjective evaluation of sutures and objective measurements based on physical tests by using fuzzy logic method. The purpose of this modeling is to predict handling properties of sutures from physical measured parameters.

\section{EXPERIMENTAL PART}

Braided nylon sutures "Polyamide T Noir" commercialized by Adhe-els were evaluated. They are made from multifilament yarns and have different USP sizes: 2 - 1/0 - 2/0 - 3/0. The size 3/0 correspond to the smallest diameter. Suture sizes are defined by the United States Pharmacopeia (U.S.P.). For a given USP size, the diameter of thread differs depending on the 
suture material class (Absorbable, Non-absorbable...). Sutures diameters are mentioned in table 1.

Table 1. Sutures diameters measured with microscope

\begin{tabular}{lcccc}
\hline Size (USP) & $\mathbf{2}$ & $\mathbf{1 / 0}$ & $\mathbf{2 / 0}$ & $\mathbf{3 / 0}$ \\
\hline Diameter (mm) & 0.782 & 0.469 & 0.421 & 0.342 \\
\hline
\end{tabular}

\subsection{Objective evaluation}

In order to evaluate knot security, friction coefficient, bending rigidity and compressibility can be used $[2,8]$. We determined friction coefficient by twist method proposed by Lindberg and Gralen and adopted by Gupta [8]. An experimental device composed of six pulleys was developed and fixed to the lower jaws of a tensile tester. Two identical sutures are fixed to the upper jaws and passed around pulleys and two identical masses are attached to the two sutures as shown in figure 1 .

Equation 1 allows the calculation of friction coefficient $\mu$ :

$$
\mu=\log \left(\mathrm{T} / \mathrm{T}_{0}\right) / \pi \mathrm{n} \beta
$$

The variables $T, T_{0}, n$ and $\beta$ are respectively friction force registered by the load cell, normal force, number of twists and angle between sutures.

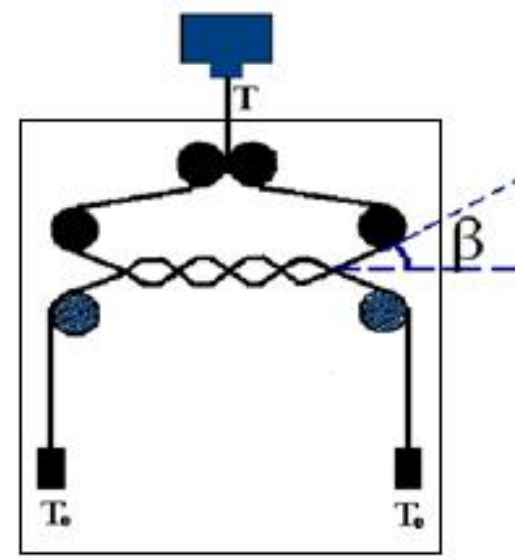

Fig.1. Schematic presentation of the developed device for the measurement of friction coefficient 
$\mathrm{T}_{0}$ was equal to $0.2 \mathrm{~N}$ and $\mathrm{n}$ is equal to 1 corresponding to a single twist simulating a surgical knot. The test was performed at a speed of $12.7 \mathrm{~mm} / \mathrm{min}$ with a load cell of $10 \mathrm{~N}$. When the test starts, sutures are tightened, then slip, producing a stick-slip phenomena. The forcedisplacement curve provided by the test shows maximum and minimum peaks corresponding to maximum and minimum friction coefficients levels. Gupta [8] considered the difference between these levels $\left(\mathrm{s}^{-} \mathrm{k}\right)$ as the magnitude of stick-slip phenomena reflecting the ease or difficulty encountered by the suture to slip in the knot.

Available literature related textile flexure concerns mainly fabrics and fibers. Today, there are no conventional methods or standards for the measurement of yarns bending rigidity. Ghane et al. [9] showed that we can assimilate yarn to a beam and determine the flexural rigidity using a two-support beam system and classic elastic equations in the case of small deflection. The yarn is fixed at one end, supported at the other and loaded in middle to measure deflection. We tried this method but results were not reproducible in the case of our braids. Inspired by this method, we had the idea to fix the two ends of sutures in order to avoid random slip of suture and apply a force $\mathrm{F}$ in the middle of the suture as shown in figure 2 . The distance 1 between two supports is $6 \mathrm{~cm}$. The deflection $\mathrm{f}$ is measured and bending stiffness $\mathrm{EI}_{\mathrm{Gz}}$ is calculated from equation 2.

$$
\mathrm{f}=\mathrm{F} \cdot \mathrm{l}^{3} /\left(192 \mathrm{E} \cdot \mathrm{I}_{\mathrm{Gz}}\right)
$$

The determination of compression behavior of the suture requires the study of compressive stresses occurring inside the knot in order to evaluate important parameters such as knot security. During, this study, compressibility of the suture was measured by determining the lateral deformation of the suture at different pressures. This deformation corresponds to the difference between the suture diameter measured with the microscope and image processing and diameter under pressure measured with thickness measurement gauge (Figure 3). Five samples from each diameter were tested in each test. 


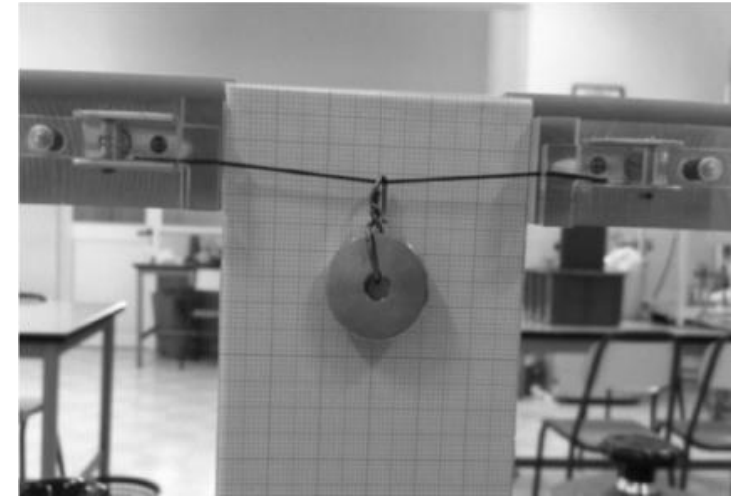

Fig.2. The two-support beam device

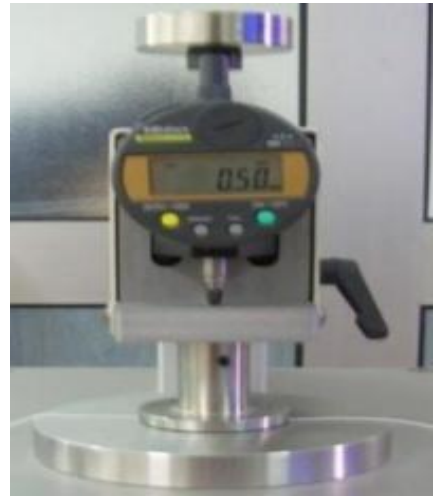

Fig.3. Thickness gauge

\subsection{Subjective evaluation}

In order to evaluate sutures subjectively we did a survey of 15 surgeons. Every surgeon was request to use our sutures in surgical operations and assign a score to the knot security which varies between -2 and 2 . The score -2 corresponds to a very low knot security and 2 corresponds to an excellent knot security as shown in figure 4.

The influence of the tying technique on the knot security was reported by many studies [2, 3, 10]. The highest knot security was found with square knots. We performed tests with this knot type (Figure 5).

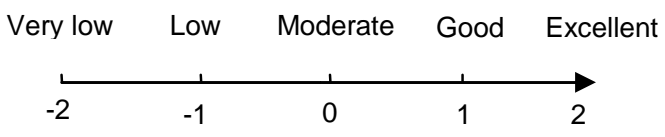

Fig.4. Evaluation criteria

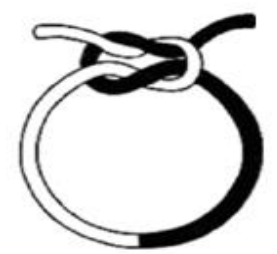

Fig.5. Square knot

\subsection{Modeling the relationship between objective tests and subjective evaluation}

Subjective or sensory evaluation gives an idea about surgeon perception towards sutures handling characteristics but it is difficult to use because of the lack of standards describing interpretation of human evaluation. On the other side, objective tests provide precise quantitative data which describe sutures hand. In literature, some researchers studied the relationship between objective tests and subjective evaluation mainly in the case of knitted fabric hand [11-13].

The prediction of knot security from objective tests requires correlation between subjective evaluation of handling characteristics and physical parameters obtained from objective tests. 
Relationship between physical parameters and knot security is complex. For the correlation, we used fuzzy logic technique which has been often used in the field of fabric hand evaluation [14-17]. This method has shown many advantages in characterizing some complex concepts related to sensory and instrumental evaluation of hand properties. Fuzzy logic is flexible and tolerant of imprecise data that can model non linear functions. A fuzzy Inference system can be described via the diagram shown in figure 6 .

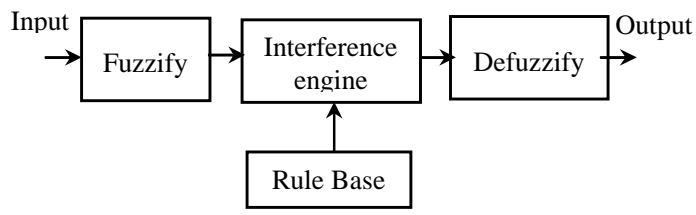

Fig.6. Diagram of Fuzzy Interference System

In our case, the inputs are physical parameters and the output is the score attributed by the surgeon. We used the Java Eclipse software to program Fuzzy Interference System.

\section{RESULTS AND DISCUSSIONS}

\section{- Objective evaluation}

Figure 7 presents an example of force-displacement diagrams registered by the tensile tester according to the twist method. This diagram shows maximum and minimum peaks. These peaks were used to calculate the term $\left(s_{-}-k\right)$ corresponding to the difference between successive friction coefficients by using a program developed on Matlab software.

Figure 8 shows mean values of these differences for different sutures sizes. Confidence intervals are calculated with an error margin of $5 \%$. 


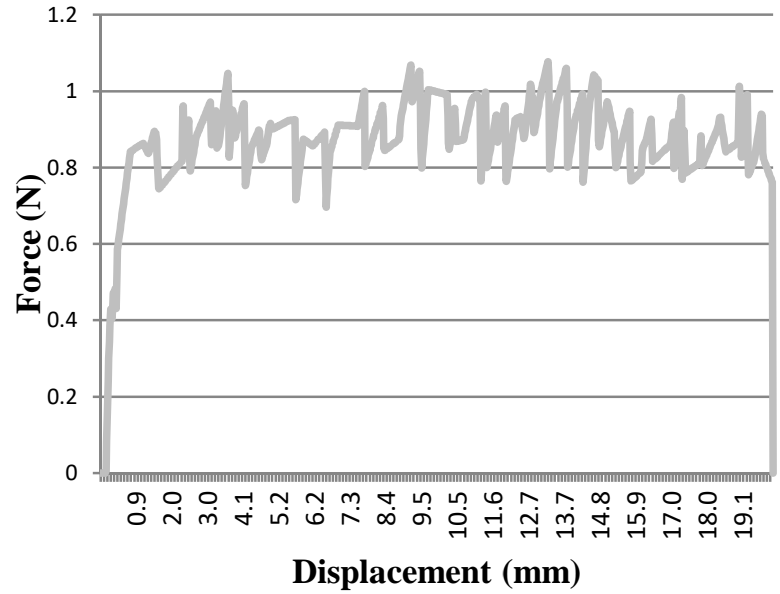

Fig.7. Example of force-displacement diagram

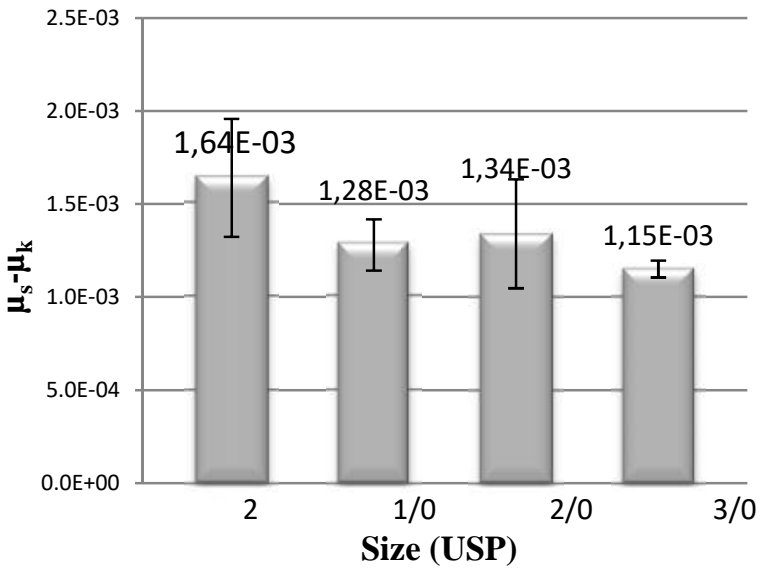

Fig.8. Variation of $s^{-}{ }_{k}$ with suture diameter

As can be seen from figure 8 , friction coefficient is overall proportional to suture diameter. Suture having the highest diameter (USP 2) shows the highest friction coefficient. This is explained by the fact that when the diameter increases, filaments are more inclined relatively to braid axis and are more opposed to tangential movement of braid surface. During suture slippage tensile tester load cell records consecutive maximum and minimum forces which are different.

Figure 9 shows results of bending rigidity test. It is quite obvious that when the diameter of the braid increases, the bending stiffness increases. Sutures having low bending rigidities are more flexible and allow easy handling by the surgeon.

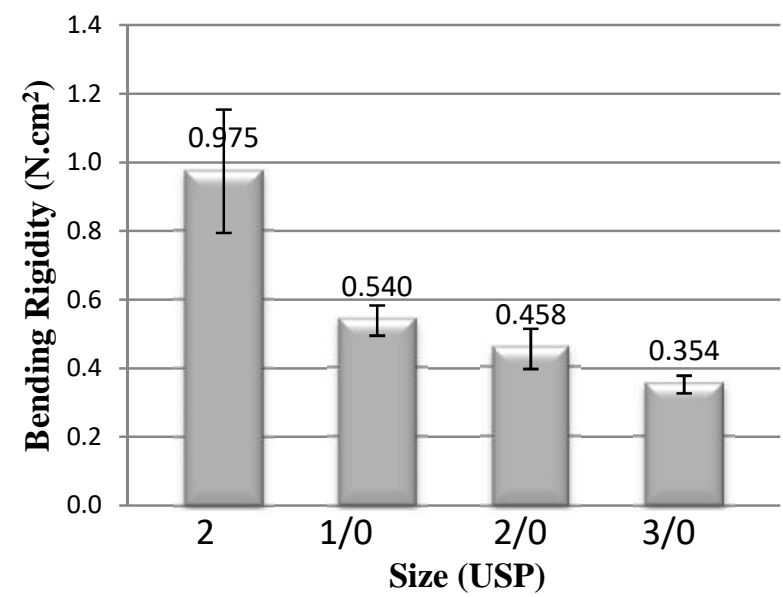

Fig.9. Variation of bending rigidity with suture diameter

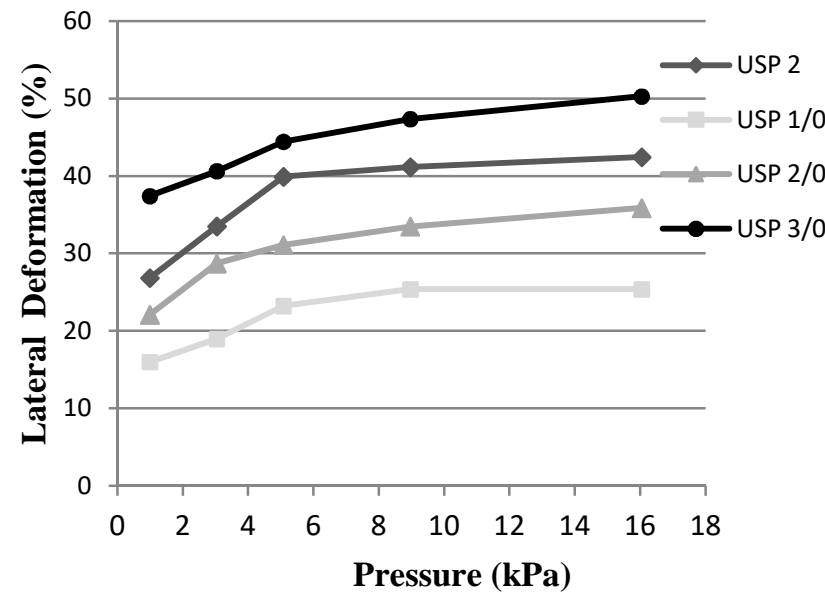

Fig.10. Lateral deformation of sutures under different pressures 
For sutures compressibility evaluation, we determined sutures diameters by using a microscope and image processing (Table 1), then, lateral deformation with the following pressures $(\mathrm{kPa}): 1$ - 3.05 - 4.2 - 5.09 - 8.97 - 16.05. Results are shown in figure 10. We can see that suture have the smallest diameter (USP 3/0) has the highest lateral deformation.

\section{- Subjective evaluation}

We collected scores attributed by surgeons for the different sutures used in different surgeries (Table 2). Results are presented in term of percentage relatively to the total number of evaluations. We can notice that sutures which have low diameter (USP 2/0 and 3/0) present good knot security.

Table 2. Results of the surgeons sensory evaluation

\begin{tabular}{lcccc}
\hline Size (USP) & $\mathbf{2}$ & $\mathbf{1 / 0}$ & $\mathbf{2 / 0}$ & $\mathbf{3 / 0}$ \\
\hline $\begin{array}{l}\text { Very Low } \\
(\%)\end{array}$ & 13 & 0 & 0 & 0 \\
Low (\%) & $\mathbf{5 0}$ & 19 & 0 & 0 \\
Moderate (\%) & 37 & $\mathbf{5 6}$ & 11 & 25 \\
Good (\%) & 0 & 19 & $\mathbf{7 8}$ & $\mathbf{5 0}$ \\
Excellent (\%) & 0 & 6 & 11 & 25 \\
\hline
\end{tabular}

Table 3. Numeric data of inputs and outputs of the model

\begin{tabular}{|c|c|c|c|c|}
\hline \multirow[b]{2}{*}{ 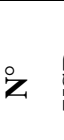 } & $\mathbf{E}_{1}$ & $\mathbf{E}_{2}$ & $\mathbf{E}_{3}$ & Output \\
\hline & $\left(\mathrm{N} . \mathrm{cm}^{2}\right)$ & $(\%)$ & $(\mu \mathrm{s}-\mu \mathrm{k})$ & $\begin{array}{l}\text { Knot } \\
\text { security }\end{array}$ \\
\hline 2 & 0.975 & 26.85 & $1.64 \mathrm{E}-03$ & -1 \\
\hline $1 / 0$ & 0.54 & 15.99 & $1.28 \mathrm{E}-03$ & 0 \\
\hline $2 / 0$ & 0.458 & 22.09 & $1.34 \mathrm{E}-03$ & 1 \\
\hline $3 / 0$ & 0.354 & 37.43 & $1.15 \mathrm{E}-03$ & 1 \\
\hline
\end{tabular}

\section{- Development of the fuzzy-logic based model for correlating objective tests and knot} security

The table 3 summarizes the numeric data of inputs and outputs of the model.

$E_{1}, E_{2}$ and $E_{3}$ are respectively bending rigidity, lateral deformation (under a pressure of $1 \mathrm{kPa}$ ) and friction coefficient.

A fuzzy inference system contains four components: the fuzzifier, inference engine, rule base and defuzzifier (Figure 6) [13, 16]. The fuzzifier maps the inputs numbers into corresponding fuzzy memberships in order to activate rules that are in terms of linguistic variables. Then, the fuzzifier determines for a given input the degree of membership to the fuzzy sets via the membership functions.

The rule base contains linguistic rules provided by experts. In our case, there are no experts able to provide rules which describe the relationship between physical parameters and knot security of sutures. So, we have to extract the rules of the model from numerical data. 
The inference engine consists in passing from input fuzzy sets to output fuzzy ones. Every activated rule has an output fuzzy set using fuzzy operators. It is possible that one or more rules are kept out. The used method for the implication is the minimum. Then, all rules are aggregated to obtain only one output set.

To defuzzify is to return a number from the output fuzzy set. There are several methods used for defuzzification but the most used is the centroid. It calculates and returns the center of gravity of the aggregated fuzzy sets.

For the extraction of rules, we used the method of clustering map [18, 19]. From Table 3, we obtain Figure 11 which correspond to the mapping of each two mechanical parameters together.
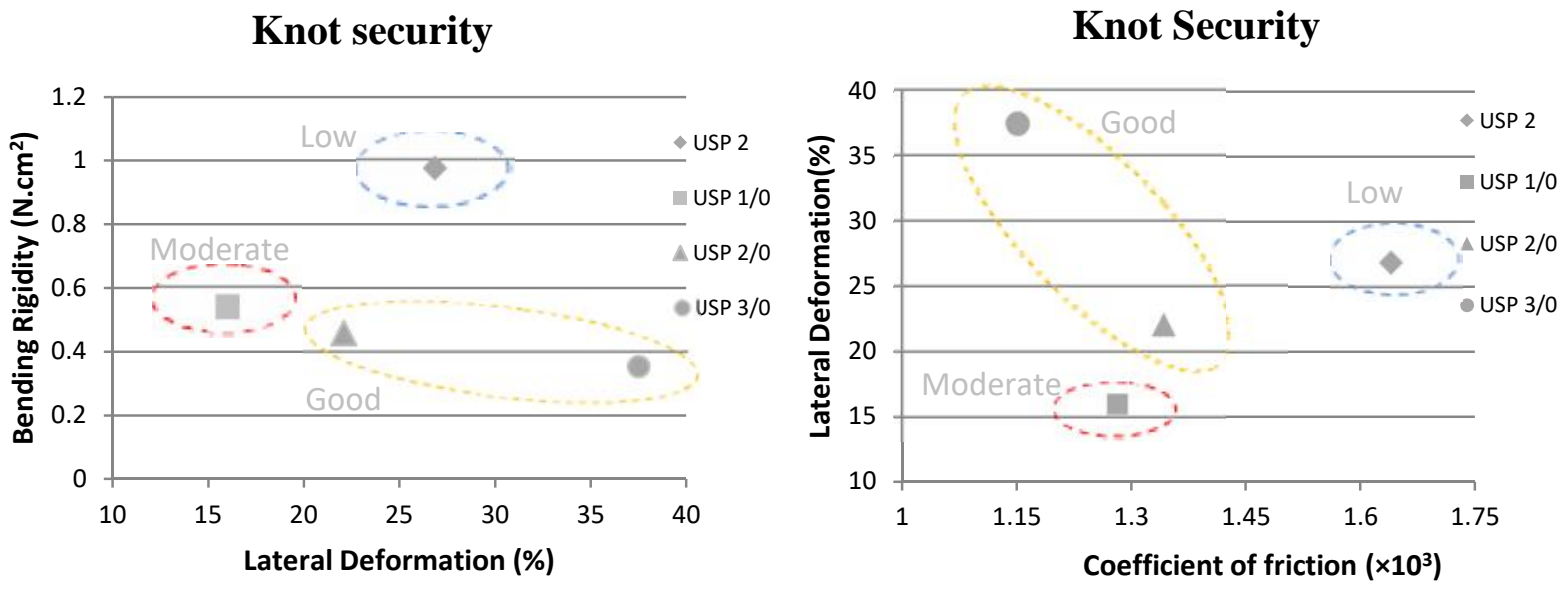

Fig.11. Mapping of the inputs: $E_{1}$ and $E_{2}$ (left), $E 2$ and $E_{3}$ (right)

The obtained maps show 3 clusters: Low, Moderate and Good. Our sutures were divided into 3 subsets and the following rules were extracted.

\section{Rules extracted from objevtive and subjective data}

Cluster1: If $0.4 \leq \mathrm{E}_{1} \leq 0.7 \& 15 \leq \mathrm{E}_{2} \leq 20 \quad \& 1.25 \leq \mathrm{E}_{3} \leq 1.35$ then knot security is Moderate

Cluster2: If $0.8 \leq \mathrm{E}_{1} \leq 1.2 \& 25 \leq \mathrm{E}_{2} \leq 30 \quad \& \quad 1.6 \leq \mathrm{E}_{3} \leq 1.7$ then knot security is Low

Cluster3: If $0.2 \leq \mathrm{E}_{1} \leq 0.6 \& 20 \leq \mathrm{E}_{2} \leq 40 \quad \& \quad 1 \leq \mathrm{E}_{3} \leq 1.45$ then knot security is Good

\section{Fuzzy Inference System}

The steps of fuzzy modeling procedure are described as following: 
- Step 1: Fuzzification of input variables

Based on Figure 11, the bending rigidity was divided into three fuzzy values named: Low, medium and high on the scale of 0.2-1.2 N.cm ${ }^{2}$ (Figure 12). The scale of physical parameters was decided according to minimum and maximum values of these parameters among our samples.

The lateral Deformation was divided into four fuzzy values named: Very Low, low, medium and high (Figure12).By referring to figure 11 the coefficient of friction ( $\mu \mathrm{s}-\mu \mathrm{k}$ ) was divided into four fuzzy values named: Low, medium and high (Figure12).

- Step 2: Fuzzification of output variable

The sensory scores were divided into five fuzzy values as shown in figure 12 .
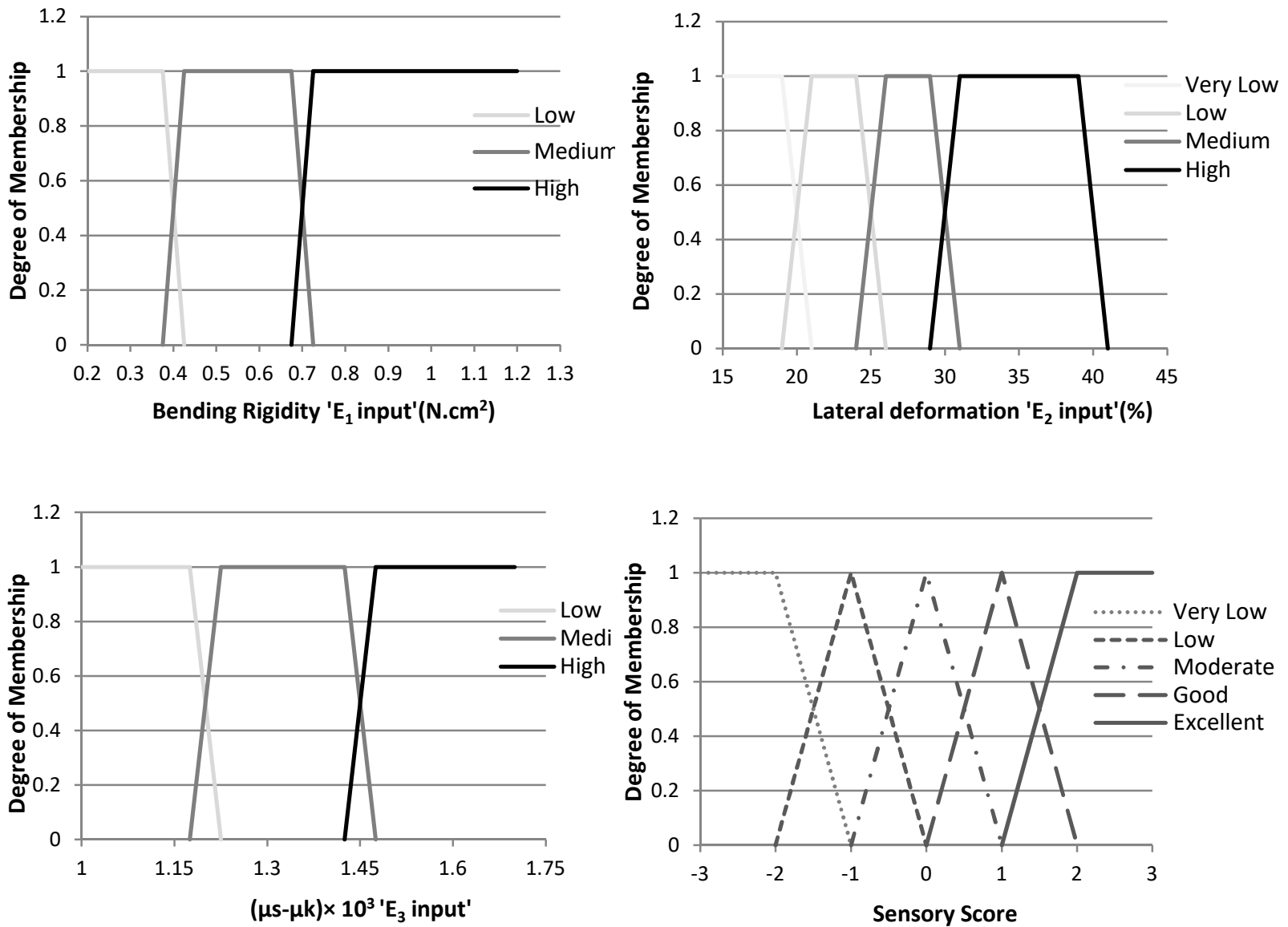

Fig.12. Membership functions of the different inputs and the output

- Setp 3: Reformulation of the rules 
After determination of membership functions, we reformulate the rules linguistically in order to be used later in the programming.

If $\mathrm{E}_{1}$ is medium $\& \mathrm{E}_{2}$ is very low $\boldsymbol{\&} \mathrm{E}_{3}$ is medium then knot security is Moderate.

If $\mathrm{E}_{1}$ is high $\boldsymbol{\&} \mathrm{E}_{2}$ is medium $\boldsymbol{\&} \mathrm{E}_{3}$ is high then knot security is low.

If $\mathrm{E}_{1}$ is low $\boldsymbol{\&} \mathrm{E}_{2}$ is high $\boldsymbol{\&} \mathrm{E}_{3}$ is low then knot security is good.

If $\mathrm{E}_{1}$ is medium $\boldsymbol{\&} \mathrm{E}_{2}$ is low $\boldsymbol{\&} \mathrm{E}_{3}$ is medium then knot security is good.

The Mandami method was used to aggregate these rules and compute the output from input values.

- Step 4: Defuzzification of output

This goal of this step is to calculate a score by calculating the center of gravity of the output fuzzy set.

The fuzzy model calculates the score related to knot security from the three entered physical data.

\section{Fuzzy system programming}

In the beginning of the program, we have to declare input and output variables and enter input and output membership functions described in the fuzzification step. Then we introduce rules describing used fuzzy operators. We use the operator 'minimum' to calculate the intersection between predicates and activate rules; we used the operator 'maximum' to aggregate or accumulate rules. The transition from the fuzzy "world" to the real "world", requires the calculation of the center of gravity of output fuzzy set.

Figure 13 gives an overview of the calculation program. We can see that, for a suture non evaluated by surgeons having a bending rigidity of $0,69 \mathrm{~N} . \mathrm{cm}^{2}$, a lateral deformation of $20 \%$ and a coefficient of friction of 1,43 E-03 (E1=0.69, E2=20, E3=1.43), the output Knot_security is the aggregate output fuzzy set built by the aggregation of fuzzy sets obtained by each rule. The calculation of the center of gravity of the output fuzzy set leads to a Knot_security equal to 0.5 . 


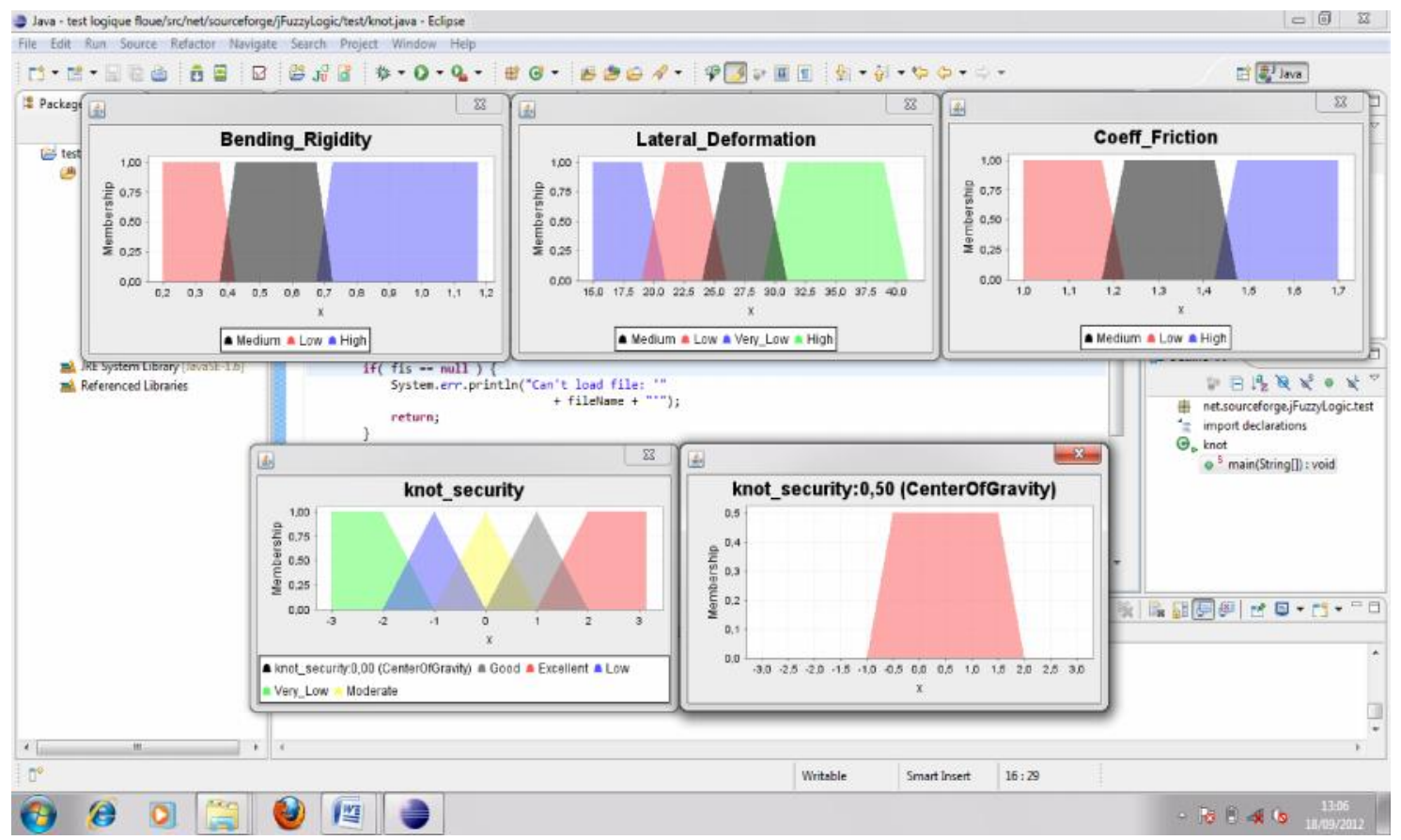

Fig.13. Overview of the calculation program interface

The output equal to 0.5 obtained after defuzzification means that we have a suture having a moderate to good knot security. This same suture was then evaluated by surgeons; half of them assigned a 0 as a score and the other half a score of 1 for the knot security. Finally, we can say that developed Fuzzy-logic method and subjective evaluation done by the surgeons converge to similar results.

\section{CONCLUSION}

In this work, we propose an objective method to evaluate knot security of braided sutures made of nylon multifilament yarns. Knot security is a handling characteristic which can be evaluated only subjectively by surgeons during implantation. The developed method is based on a fuzzy model which correlates physical parameters determined by objective tests and knot security of sutures evaluated by surgeons.

We showed that it is possible to reproduce, reliably, surgeons appreciation of sutures. In fact, we developed a fuzzy model allowing the prediction of knot security from three physical parameters of the suture: bending rigidity, lateral deformation and friction coefficient.

Further work will focus on the evaluation of knot security of other sutures available in the market in order to generalize results obtained in this work. 


\section{REFERENCES}

[1] Tremblay S, and Mantovani D. Les fils de suture : de fil en aiguille. Le Médecin du Québec, 2003 38(10), 105-108.

[2] Chu C.C .Classification and General Characteristics of Suture Materials, Chapter 4. In C.C Chu, J. A Fraunhofer, and H.P. Greisler (Eds),Wound Closure: Biomaterials and Devices. CRC Press, 1997, pp. 39-64, ISBN 0-8493-4964-8.

[3] Fraunhofer J.A, and Chu C.C. Mechanical properties, Chapter 6. In: C.C Chu, J. A Fraunhofer, and H.P. Greisler (Eds), Wound Closure: Biomaterials and Devices. CRC Press 1997, pp. 107130, ISBN 0-8493-4964-8.

[4] Herman J.B. Tensile strength and knot security of surgical suture materials. Am.Surg., 1971,37, 209-217.

[5] Lo I.K. Y, Burkhart S. S, Chan K.C and Athanasiou K. Arthroscopic knots: determining the optimal balance of loop security and knot security. The Journal of Arthroscopic and Related Surgery, 2004, 20(5), 489-502.

[6] Hassinger S. M, Wongworawat M. D and Hechanova J. W. Biomechanical characteristics of 10 arthroscopic knots. The Journal of Arthroscopic and Related Surger, 2006, 22 (8), 827-83.

[7] Ben Abdessalem S, Debbabi F, Jedda H, Elmarzougui S and Mokhtar S. Tensile and Knot Performance of Polyester Braided Sutures. Textile Research Journal, 2009, 79(3), 247-252.

[8] Gupta B.S. Frictional Properties of Textile Materials. In: Pastore C. M., and Kiekens P (Eds), Surface characteristics of fibers and textiles. New York, Les Etats Unis: Marcel Dekker, 2001, pp.59-92. ISBN 0824700023

[9] Ghane M, Sheikhzadeh M, Halabian A. M. and Khaburi S. Bending Rigidity of Yarn Using a Two Supports Beam System. FIBRES \& TEXTILES in Eastern Europe, 2008, 16 (3).

[10] Debbabi F, Ben Abdessalem S, and Limem S. New test methods to evaluate the performance of dermatological braided sutures from both the doctor and the patient sides. Journal of Textile Institute, 2011, 102(6), 548-557.

[11] Park S.W, Hwang Y.G, Kang B.C, and Yeo S.W. Applying Fuzzy Logic and Neural Networks to Total Hand Evaluation of Knitted Fabrics. Textile Research journal, 2000, 70(8), 675-681.

[12] Jeguirim S.G, Sahnoun M, Babay D.A, Cheikhrouhou M, Schacher L.M, Adolphe D.C. Predicting compression and surfaces properties of knits using fuzzy logic and neural networks techniques. International Journal of Clothing Science and Technology. 2011,23(5), 294-309.

[13] Agarwal G, Koehl L, and Perwuelz A. Interaction of wash-ageing and useof fabric softener for drapeability of knitted fabrics. Textile Research Journal, 2011, 81(11), 1100-1112.

[14] Raheel M. and Liu J. An Empirical Model for Fabric Hand: Part I: Objective Assessment of Light Weight Fabrics. Textile Research Journal, 1991, 61(31), 31-38. 
[15] Zeng X. and Koehl L. Representation of the Subjective Evaluation of the Fabric Hand Using Fuzzy Techniques. International Journal Of Intelligent Systems, 2003 18, 355-366.

[16] Agarwal G, Koehl L, Perwuelz A, and Lee K.S. Interaction of Textile Parameters, Wash-ageing and Fabric Conditioner with Mechanical Properties and Correlation with Textile-hand. II. Relationship between Mechanical Properties and Textile-hand. Fibers and Polymers , 2011,12 (6), 795-800.

[17] Jeguirim S.G, Adolphe D.C, Sahnoun M, Babay D.A, Schacher L.M, Cheikhrouhou M. Intelligent Techniques for Modeling the Relationships between Sensory Attributes and Instrumental Measurements of Knitted Fabrics. Journal of Engineered Fibers and Fabrics, 2012,7 (3), 88-97.

[18] Agarwal G. Interaction of textile parameters, wash ageing and use of fabric softener during the laundry with mechanical properties of the knitted fabrics and correlation with textile hand. 2011. Ph.D. Thesis, University of science and Technology, Lille1, 206 p.

[19] Zhu Y. Contribution à l'évaluation et à la modélisation du bien-être des matériaux textiles habillement par l'utilisation des techniques de calcul avancé. 2010, Ph.D Thesis, Universitey of Lille 1, 262 pages.

How to cite this article:

Turki S and Ben Abdessalem S. Development of an objective method for the assessment of nylon sutures knot security. J. Fundam. Appl. Sci., 2017, 9(2), 988-1000. 